\title{
Recent Developments and Analysis of Electromagnetic Metamaterial with all of its Application in Terahertz range
}

\author{
Avik Chakraborty \\ Assistant Professor, Electronics \&Communication Engg. Deptt., Bengal Institute of Technology \&Management \\ .Bolpur, Birbhum, Contact Address: Senpara, Kalitala Road,Po+Dist-Jalpaiguri,PIN-735101.
}

\begin{abstract}
Recent advances in metamaterials (MMs) research have highlighted the possibility to create novel devices with electromagnetic functionality. The metamaterial have the power which can easily construct materials with a user-designed EM response with a particular target frequency. This is the important phenomena of THz frequency region that can make a considerable progress in design fabrication, and define the characteristics of MMs at $\mathrm{THz}$ frequencies. This article illustrates the latest advancements of $\mathrm{THz} M \mathrm{MM}$ research.
\end{abstract}

Key word: Metamaterials (MMs), Terahertz $(\mathrm{THz})$.

\section{Introduction:}

Recently artificially structured electromagnetic (EM)materials have become an extremely active research area because of the possibility of creating materials which exhibit novel EM response which is not available in natural materials. This includes negative refractive index,superlensing, cloaking,and more generally ,coordinating transformation materials. [1]

The EM response for the MMs originates from oscillating electrons in highly conducting metals such as gold or copper allowing for a designed specific resonant response of the electrical permittivity $\left(€=\epsilon_{1}+i \epsilon_{2}\right)$ or magnetic permeability $\left(\mu=\mu_{1}+i \mu_{2}\right)$. In artificial materials negative $\mu_{1}$ was first realized with split ring resonators (SRRs).SSRs are composed of metallic rings with gaps which was theoretically introduced by Pendry et al.[2],and experimentally verified by Smith et al.[3]in 2000.

Since the EM response of MMs can be designed over a large portion of the EM spectrum by ,to first order, simply scaling the dimensions of the structures.MMs have played an increasingly important role particularly in the construction of functional $\mathrm{THz}$ devices .For THz MMs, the unit cell is few tens of microns .For these length scales, conventional micro -fabrication techniques offer considerable flexibility to experimentally realize novel MM structures and devices.

In another way it can be stated that it is an artificial materials engineered to have properties that may not be found in nature.Metamaterials usually gain their properties from structure rather than composition, using small in homogeneities to create effective macroscopic behavior.[1]

The primary research in metamaterials investigates materials with negative refractive index .Negative refractive index materials appear to permit the creation of superlenses which can have a spatial resolution below that of the wavelength. In other word a form of invisibility has been demonstrated at least over a narrow wave band with gradient index materials. Although the first metamaterials were electromagnetic [4 ],acoustic and seismic metamaterials are also areas of active research.[5],[6]

Potential applications of metamaterials are diverse and include remote aerospace applications, sensor detection and infrastructure monitoring, smart solar power management ,public safety,radomes,high frequency battlefield communication, and lenses for gain antennas, improving ultrasonic sensors, and even shielding structure from earthquakes.

\footnotetext{
A brief idea about SPLIT -RING RESONATORS (SRRs)

SRRs are composed of metallic rings with gaps. Split ring resonator(SRR)is a component part of a Negative Index Metamaterial(NIM),also known as Double Negative Metamaterials (DNG) or Left Handed Medium(LHM).They are also component part of other types of metamaterials such as Single Negative Metamaterial (SNG).SRRs are also used for research in Terahertz metamaterials ,Acoustic metamaterials,and Metamaterial antennas. A single cell SRR has a pair of enclosed loops with splits in them at opposite ends. Due to splits in the rings the structure can support resonant wavelengths which is much larger than the diameter of the rings. This would not happen in closed rings. The small gaps between the rings produces large capacitance values which lower the resonating frequency, as time constant is large .The dimensions of the structure are small compared to the resonant wavelength. This results in low radiative losses, and very high quality factors.
} 


\section{Configuration wise SPLIT-RING RESONATORS (SRRs)}

There is a variety of split -ring resonators -rod-split rings, nested split-rings, single split rings, deformed split rings, spiral split rings, and extended $\mathrm{S}$ - structures. The variations of split ring resonators have achieved different result, including smaller and higher frequency structures. The research which involves some of these types are discussed throughout the article. To date (December 2009) the capability for desired results in visible spectrum has not been achieved. However in 2005 , it was noted that physically a nested circular split ring resonator must have an inner radii of 30 to 40 nanometer has achieved success in the mid range of the visible spectrum.

\section{General configuration of SPLIT- RING RESONATOR (SRRs):}

SRRs are one of the most common elements used to fabricate metamaterials. Split -ring resonators are non magnetic materials, which are usually fabricated from circuit board material to create metamaterials. At first a single SRR looked like a ring with small segment removed which result in a " $c$ " shape, on fiberglass, printed circuit board material. In this type of configuration it is actually two concentric bands of non magnetic conductor material. There is one gap In each band placed 180 degree relative to each other. The gap in each band gives it the distinctive "c" shape ,rather than a totally circular or square shape. Then multiple cells of this double band configuration are fabricated into circuit board material by an etching technique and lined with copper wire strip arrays are added. After processing, the boards are cut and assembled into an interlocking unit. It is constructed into a periodic array with a large number of SRRs. SRRs were first used to fabricate Left Handed Metamaterials(LHM) for the microwave range, and several years later for the terahertz range . By 2007 SRRs have been used for research in acoustic metamaterials.

\section{Types of SPLIT-RING RESONATORS:}

In research based in metamaterials and specifically negative refractive index , there are different types of split ring resonators. Of the example mentioned below all of them have a gap in each ring .In other words, with a double ring structure, each ring has a gap.[7]

1) 1-D Split -Ring Structure with two square rings, one inside the other .One set of cited "unit cell" dimensions would be an outer square of $2.62 \mathrm{~mm}$ and an inner square of .25mm. 1-D structures such as this are easier to fabricate compared with constructing a rigid 2-D structure.[7]

2) Symmetrical -Ring Structure is another classic example .Described by the nomenclature these are two rectangular square D type configurations, exactly the same size, laying flat, side by side, in the unit cell. Also these are not concentric .One set of cited dimensions are $2 \mathrm{~mm}$ on the shorter side and $3.12 \mathrm{~mm}$ on the longer side .The gaps in each ring face each other in the unit cell.

3) Omega Structure as the nomenclature describes, it has an $\Omega$ shaped ring structure. There are two of these, standing vertical, side by side, instead of lying flat in the unit cell.[7]

\section{Electromagnetic metamaterials}

Metamaterials have become a new sub discipline within physics and electromagnetism (especially optics and photonics.

They show promise for optical and microwave applications such as new types of beam steerers, modulators, band-pass filters, lenses, microwave couplers, and antenna systems. Furthermore, the lower density of materials means that components, devices, and systems can be lightweight and small, while at the same time enhancing system and component performance.

Metamaterials consist of periodic structures. Electromagnetic metamaterial affects electromagnetic waves by having structural features smaller than the wavelength of light. In addition, if a metamaterial is to behave as a homogeneous material accurately described by an effective refractive index, its features must be much smaller than the wavelength. To date, sub wavelength structures have shown only a few questionable results at visible wavelengths.

For microwave radiation, the structures need only be on the order of few centimeters. Microwave frequency metamaterials are usually synthetic, constructed as arrays of electrically conductive elements (such as loops of wire) which have suitable inductive and capacitive characteristics. These are known as split-ring resonators.

Another structure which can exhibit sub wavelength characteristics are frequency selective surfaces (FSS) known as Artificial Magnetic Conductors (AMC) or alternately called High Impedance Surfaces (HIS). These also have inductive and capacitive characteristics, which are directly related to its sub wavelength structure.

Photonic crystals and frequency-selective surfaces such as diffraction gratings, dielectric mirrors, and optical coatings do have apparent similarities to sub wavelength structured metamaterials. However, these are usually 
considered distinct from sub wavelength structures, as their features are structured for the wavelength at which they function, and thus cannot be approximated as a homogeneous material.

However, novel-material structures such as photonic crystals are effective with the visible light spectrum. The middle of the visible spectrum has a wavelength of approximately $560 \mathrm{~nm}$ (for sunlight), the photonic crystal structures are generally half this size or smaller, that is $<280 \mathrm{~nm}$.

\section{Classification of Electromagnetic Metamaterials}

Various types of composite material, both electromagnetic and other types are being studied by various research groups worldwide (see all sections and references below). Electromagnetic metamaterials are represented by different classes, as follows:

\section{Negative index materials}

In negative index metamaterials (NIM), both permittivity and permeability are negative resulting in a negative index of refraction. Hence, because of the double negative parameters these are also known as Double Negative Metamaterials or double negative materials (DNG) Other terminologies for NIMs are "left-handed media", "media with a negative refractive index", and "backward-wave media", along with other nomenclatures.

In optical materials, if both permittivity $\boldsymbol{\varepsilon}$ and permeability $\boldsymbol{\mu}$ are positive this results in propagation in the forward direction. If both $\varepsilon$ and $\mu$ are negative, a backward wave is produced. If $\varepsilon$ and $\mu$ have different polarities, then this does not result in wave propagation. Mathematically, quadrant II and quadrant IV have coordinates $(0,0)$ in a coordinate plane where $\varepsilon$ is the horizontal axis, and $\mu$ is the vertical axis.

In 1968 Victor Veselago published a paper theorizing plane wave propagation in a material whose permittivity and permeability were assumed to be simultaneously negative. In such a material, he showed that the phase velocity would be anti-parallel to the direction of poynting vector. This is contrary to wave propagation in natural occurring materials. In the years 2000 and 2001, papers were published about the first demonstrations of an artificial material that produced a negative index of refraction. By 2007, research experiments which involved negative refractive index had been conducted by many groups.

To date, materials exhibiting a negative index of refraction have only been demonstrated as artificially constructed materials.

\section{Single negative metamaterials}

In single negative (SNG) metamaterials either permittivity or permeability are negative, but not both. These are ENG metamaterials and MNG metamaterials discussed below. Interesting experiments have been conducted by combining two SNG layers into one metamaterial. These effectively create another form of DNG metamaterial. A slab of ENG material and slab of MNG material have been joined to conduct wave reflection experiments. This resulted in the exhibition of properties such as resonances, anomalous tunneling, transparency, and zero reflection. Like Negative index materials, SNGs are innately dispersive, so their permittivity $\varepsilon$, permeability $\mu$, and refraction index $\mathrm{n}$, will alter with changes in frequency.

$>$ Epsilon negative media (ENG) - permittivity $\varepsilon$ is negative while permeability $\mu$ is positive. Many plasmas exhibit this characteristic. For example noble metals such as gold or silver will exhibit this characteristic in the infrared and visible spectrums.

$>$ Mu-negative media (MNG) - permittivity $\varepsilon$ is positive while permeability $\mu$ is negative. A material, which called gyrotropic or gyromagnetic exhibits this characteristic. A gyrotropic material is a medium that has been altered by the presence of a quasistatic magnetic field. This results in the magneto-optic effect. A magneto-optic effect is any one of a number of phenomena in which an electromagnetic wave propagates through a medium that has been altered by the presence of a quasistatic magnetic field. In such a material, left- and right-rotating elliptical polarizations can propagate at different speeds, leading to a number of important phenomena. When light is transmitted through a layer of magneto-optic material, the result is called the Faraday effect: the plane of polarization can be rotated, forming a Faraday rotator. The results of reflection from a magneto-optic material are known as the magneto-optic Kerr effect (not to be confused with the nonlinear Kerr effect). Two gyrotropic materials with reversed rotation directions of the two principal polarizations are called optical isomers.

\section{Electromagnetic band gap metamaterials}

Electromagnetic band gap metamaterials control the propagation of light. This is accomplished with either a class of metamaterial known as photonic crystals (PC), or another class known as left-handed materials (LHM) Both are a novel class of artificially engineered structure, and both control and manipulate the propagation of electromagnetic waves (light). PCs can prohibit light propagation altogether. However, both the PC and LHM are capable of allowing it to propagate in certain, designed directions, and both can be designed to have electromagnetic bandgaps at desired frequencies. 
In addition, metamaterials such as Photonic crystals (PC) are complex, periodic, materials and are considered to be electromagnetic bandgap material. However, a PC is at first distinguished from sub-wavelength structures, such as tunable metamaterials, because the PC derives its properties from its band gap characteristics. In addition the PC operates at the wavelength of light, compared to other metamaterials which operate as a subwavelength structure. Furthermore, the complex response of photonic crystals functions by diffracting light. In contrast, a permittivity and permeability defines metamaterials (also a complex response), which is derived from their sub-wavelength structure and diffraction must be eliminated.

The PC is also a material in which periodic inclusions inhibit wave propagation due to destructive interference from scattering from the periodic repetition. The photonic band gap property of PCs makes them the EM analog of the electronic semi-conductor crystals.

Intended material fabrication of EBGs has the goal of creating periodic, dielectric structures, with low loss, and that are of high quality. An EBG affects the properties of the photon in the same way semiconductor materials affect the properties of the electron. So, it happens that the PC is the perfect band gap material, because it allows no propagation of light. Each unit of the prescribed periodic structure acts like large scale atoms.

Electromagnetic band gap structured (EBG) metamaterials are designed to prevent the propagation of an allocated bandwidth of frequencies, for certain arrival angles and polarizations. With EBG materials new methods utilize the properties of various dielectrics to achieve better performance. A variety of geometries and structures have been proposed to fabricate the special EBG metamaterial properties. However, in practice it is impossible to build a flawless EBG device. Factors such as advances in ideas, research, testing and development, along with the prospects of significant technological solutions, have driven the development of EBG applied science.

Commercial production of dielectric EBG devices has lagged, because commercial rewards are not readily apparent. However, start-up companies are cropping up solely focused on exploiting EBG metamaterials. These metamaterials have been manufactured for frequencies ranging from a few gigahertz's $(\mathrm{GHz})$ up to several terahertz's (THz). In other words, applications have achieved fabricated media for radio frequency, microwave and mid-infrared regions. "It now appears that EBG concepts can, in many cases act as improved replacements for conventional solutions to electromagnetic problems." Applicable developments include an EBG transmission line, fabricated utilizing the special properties of metamaterials, EBG woodpiles made of square dielectric bars, and several different types of low gain antennas.

An EBG is a result of a metamaterial that functions in the regime where the period is an appreciable amount of the wavelength, and constructive and destructive interference occur.

\section{Double positive medium}

Double positive mediums (DPS) do occur in nature such as naturally occurring dielectrics. Permittivity and magnetic permeability are both positive and wave propagation is in the forward direction. Artificial materials have been fabricated which DPS, ENG, and MNG properties have combined.

\section{Bi-isotropic and bianisotropic metamaterials}

Categorizing metamaterials into double or single negative, or double positive, is normally done based on the assumption that the metamaterial has independent electric and magnetic responses described by the parameters $\varepsilon$ and $\mu$. However in many examples of electromagnetic metamaterials, the electric field causes magnetic polarization, and the magnetic field induces an electrical polarization, i.e., magnetoelectric coupling. Such media are denoted as being bi-isotropic. Media which are exhibit magneto-electric coupling, and which are also anisotropic (which is the case for many commonly used metamaterial structures), are referred to as bianisotropic. are denoted as bi-anisotropic.

Intrinsic to magnetoelectric coupling of bi-isotropic media, are four material parameters interacting with the electric $(\mathbf{E})$ and magnetic $(\mathbf{H})$ field strengths, and electric $(\mathbf{D})$ and magnetic $(\mathbf{B})$ flux densities. These four material parameters are $\varepsilon, \mu, \kappa$ and $\chi$ or permittivity, permeability, strength of chirality, and the Tellegen parameter respectively. Furthermore, in this type of media, the material parameters do not vary with changes along a rotated coordinate system of measurements. In this way they are also defined as invariant or scalar.

The intrinsic magneto electric parameters, $\kappa$ and $\chi$, affect the phase of the wave. Furthermore, the effect of the chirality parameter is to split the refractive index. In isotropic media this results in wave propagation only if $\varepsilon$ and $\mu$ have the same sign. In bi-isotropic media with $\chi$ assumed to be zero, and $\kappa$ a non-zero value, different results are shown. Both a backward wave and a forward wave can occur. Alternatively, two forward waves or two backward waves can occur, depending on the strength of the chirality parameter. 


\section{Chiral metamaterials}

When a metamaterial is constructed from chiral elements then it is considered to be a chiral metamaterial, and the effective parameter $\mathrm{k}$ will be non-zero. This is a potential source of confusion as within the metamaterial literature there are two conflicting uses of the terms left and right-handed. The first refers to one of the two circularly polarized waves which are the propagating modes in chiral media. The second relates to the triplet of electric field, magnetic field and Pointing vector which arise in negative refractive index media, which in most cases are not chiral.

Wave propagation properties in chiral metamaterials demonstrate that negative refraction can be realized in chiral metamaterials with a strong chirality, with neither negative $\varepsilon$ nor $\mu$ as a requirement. This is because the refractive index of the medium has distinct values for the left and right, given by

$\mathrm{n}=\sqrt{\epsilon \mu}+\mathrm{k}$

It can be seen that a negative index will occur for one polarization if $\kappa>\sqrt{\epsilon \mu}$. In this case, it is not necessary that either or both $\varepsilon$ and $\mu$ be negative for backward wave propagation.

\section{Metamaterial and its invisibility}

Metamaterials have significantly extended the range of electromagnetic properties available to device designers. An interesting application of these new materials is to the problem of cloaking, where the goal is to render an object invisible to electromagnetic radiation within a certain frequency range.

To be invisible in the conventional sense is to be undetectable to electromagnetic radiation within a given frequency range; light must not be scattered or absorbed. An immediate and unfortunate consequence of this requirement is that an invisible person must also necessarily be blind (in the relevant frequency range)! In many applications, partial invisibility is sufficient. An example is stealth technology, where the aim is to hide an object from radar; since conventional radar detects light reflected by an object, it would be enough either to absorb all the light, or to redirect it away from the detector(s).

\section{Techniques of metamaterial invisibility}

Three distinct ideas have emerged for metamaterial invisibility:

The first, due to Alú and Engheta, is to use a metamaterial coating to dramatically reduce the scattering from small objects. The coating must be tailored according to the object to be hidden, and is designed so that the lowest order (and hence, most significant) multipole terms in the scattered field are canceled. As the object size increases, higher-order multipoles become more important in the scattered field, and this method of cloaking becomes more difficult, although the possibility of concealing collections of particles in close proximity has been established [8], at least in theory.

The second technique employs the peculiar properties of negative-index media. These do not exist in nature, but have been made possible by the advent of metamaterials. In these materials, the real parts of the permittivity $\varepsilon$ and permeability $\mu$ are simultaneously negative. They can also be used to make super-lenses. Milton et al. showed that a polarizable object placed close to such a lens becomes effectively invisible. As the losses in the superlens material are reduced, the field scattered by the object is damped more and more, until it disappears in the lossless limit.

The third approach is the most promising, and is the only one to have been demonstrated in an experiment. It is therefore the focus of this review. It is based on the idea that a transformation of space can be mimicked by an appropriate transformation of $\varepsilon$ and $\mu$, at least as far as light is concerned. A transformation which takes a point in space and expands it to form a sphere can then be used to derive the properties of an invisibility cloak that hides any object within this sphere; to fabricate the cloak according to this recipe requires metamaterials.

Significantly, unlike the two other proposed methods, it can be used to hide objects which are much larger than the wavelength of light involved.

\section{Recent progress in THz MM Devices}

MMs play a potentially important role in creating necessary functional devices for $\mathrm{THz}$ applications. This includes perfect absorbers, THz amplitude and phase modulators, structurally reconfigurable THz MMs etc.[9]

\section{A. THz MM Absorber}

As described earlier, MMs can be regarded as an effective medium characterized by a complex electric permittivity $\varepsilon=\varepsilon_{1}+i \varepsilon_{2}$ and complex magnetic permeability $\mu=\mu_{1}+i \mu_{2}$. To create such structures, it is important to minimize losses (over the operating frequency range) associated with the imaginary portions $\left(\varepsilon_{2}\right.$ and $\left.\mu_{2}\right)$ of the effective response functions. Conversely, for many applications, it would be desirable to maximize the loss; such an absorber would be of particular importance at $\mathrm{THz}$ frequencies, where it is difficult to find naturally occurring materials with strong absorption coefficients. Tao et al. experimentally demonstrated a MM-based 
absorber with an absorptivity of 0.70 at $1.3 \mathrm{THz}$. A single unit of the absorber consists of two distinct metallic elements: an electrical ring resonator and a magnetic resonator. The electrical ring resonator consists of two single split rings sitting back to back, which couple strongly to the electric field, and negligibly to the magnetic field. Tao et al. reported a resonant MM absorber fabricated on a metallic ground plane showing an absorptivity of 0.97 at $1.6 \mathrm{THz}$. The absorber design is on a highly flexible polyimide substrate, which enables its use in nonplanar applications

\section{B. THz Quarter Waveplates}

Birefringent crystals have long been used as quarter waveplates (QWPs) in optics for converting linear polarization to circular and vice versa. The meanderline polarizer is an artificial alternative to the crystal-based QWPs for use at millimeter wave. Strikwerda et al reported two types of QWP with a center frequency of $639 \mathrm{GHz}$,one is meanderline QWP and one is electric split ring resonator based QWP,both of which are broad enough for use with continuous wave sources.

\section{THz MM Switches and Modulators}

It was proved that the $\mathrm{THz}$ switches and modulator proved to be insufficient for practical uses. After that various THz MM switches and modulators have been proposed through many modifications to the existing MMs.

Padilla et al. reported the first THz MM switch fabricated on GaAs substrates with the potential for creating dynamic MM resonance responses. Another type of $\mathrm{THz}$ modulator via an external electric bias was reported by Chen et al. The SRR structures were fabricated on a thin n-type GaAs layer, where the conductivity can be externally modified by applying a voltage bias through a group of metallic wires connecting those resonators to a voltage source. It has also been demonstrated that the phase of $\mathrm{THz}$ radiation can be modulated by a $\mathrm{THz} \mathrm{MM}$ modulator with a similar design to.

\section{Structurally Reconfigurable THz MMs}

As we know that the material properties are not only determined by nature but also depend on lattice structure, the same case is happen to MMs and to a better extent. In case of natural material the tuning of the crystal lattice is determined by the chemical bonding and nature of the atom, but in case of MMs the tenability range is more accessible because of much stronger lattice effects with proper design.

Tao et al. reported a novel structurally reconfigurable THzMM with tunable electrically and magnetically resonant responses through mechanically reorienting the micro-fabricated resonators within their unit cells.

Thus, the utilization of and implementation of MMs at $\mathrm{THz}$ frequencies holds great promise for advancing applications in this technologically relevant region of the EM spectrum. THz EM MMs have attracted enormous attention and intensive research efforts, and a number of practical MM-based $\mathrm{THz}$ devices have been developed, including filters, absorbers, QWPs, switches, and modulators.

\section{Application of metamaterials}

Below are applications of metamaterials (or types of metamaterials), which are at different stages of research.

\section{Terahertz metamaterials}

Terahertz radiation lies at the far end of the infrared band, just before the start of the microwave band.

Terahertz metamaterials are metamaterials which interact at terahertz frequencies. For research or applications of the terahertz range for metamaterials and other materials, the frequency range is usually defined as 0.1 to 10 THz. This corresponds to the millimeter and submillimeter wavelengths between $3 \mathrm{~mm}$ (EHF band) and $0.03 \mathrm{~mm}$ (long-wavelength edge of far-infrared light).

\section{Photonic metamaterials}

A Photonic metamaterial is an artificially fabricated, sub-wavelength, periodic structure, designed to interact with optical frequencies (mid-infrared). The sub-wavelength period distinguishes the photonic metamaterial from photonic band gap structures.

\section{Tunable metamaterials}

A tunable metamaterial is a metamaterial which has the capability to arbitrarily adjust frequency changes in the refractive index at will. A tunable metamaterial encompasses the development of expanding beyond the bandwidth limitations in left-handed materials by constructing various types of metamaterials. 


\section{Frequency selective surface (FSS) based metamaterial}

FSS based metamaterials have become an alternative to the fixed frequency metamaterial. The former allow for optional changes of frequencies in a single medium (metamaterial), rather than the restrictive limitations of a fixed frequency response. Other applications are also being explored.

\section{Nonlinear metamaterials}

Metamaterials may also be fabricated which include some form of nonlinear media - materials which have properties which change with the power of the incident wave. Nonlinear media are essential for nonlinear optics. However, most optical materials have a relatively weak nonlinear response, which means that their properties only change by a small amount for large changes in the intensity of the electromagnetic field. Nonlinear metamaterials can overcome this limitation, since the local electromagnetic fields of the inclusions in the metamaterial can be much larger than the average value of the field. In addition, exotic properties such as a negative refractive index, open up opportunities to tailor the phase matching conditions, which must be satisfied in any nonlinear optical structure.

\section{Metamaterial absorber}

A metamaterial absorber manipulates the loss components of the complex effective parameters, permittivity and magnetic permeability of metamaterials, to create a high electromagnetic absorber. Loss components are often noted in applications of negative refractive index (photonic metamaterials, antenna systems metamaterials) or transformation optics (metamaterial cloaking, celestial mechanics), but often not utilized in these applications.

\section{Superlens}

A superlens uses metamaterials to achieve resolution beyond the capabilities of ordinary lenses (beyond the diffraction limit). The diffraction limit is inherent in conventional optical devices or lenses.

\section{Cloaking devices}

Metamaterials are a basis for attempting to build a practical cloaking device. The proof of principle of a working invisibility cloak was demonstrated on October 19, 2006. Work continues to develop a practical cloaking device. Various theoretical models have been proposed and are being studied. A working, practical cloak is not yet available.

\section{Metamaterial antennas}

Metamaterial antennas are a class of antennas which use metamaterials to improve the performance of the antenna systems. Applying metamaterials to increase performance of antennas has garnered much interest. Demonstrations have shown that metamaterials could enhance the radiated power of an antenna. Materials which can attain negative permeability could possibly allow for properties such as an electrically small antenna size, high directivity, and tunable operational frequency.It is commercially available

\section{Acoustic metamaterials}

Acoustic metamaterials are artificially fabricated materials designed to control, direct, and manipulate sound in the form of sonic, infrasonic, or ultrasonic waves, as these might occur in gases, liquids and solids. The hereditary line into acoustic metamaterials follows from theory and research in electromagnetic metamaterials. Furthermore, with acoustic metamaterials, sonic waves can now be extended to the negative refraction domain. Control of the various forms of sound waves is mostly accomplished through the bulk modulus $\beta$, mass density $\rho$, and Chirality. The bulk modulus and density are analogies of the electromagnetic parameters, permittivity and permeability, in electromagnetic metamaterials. Related to this is the mechanics of sound wave propagation in a lattice structure. Also materials have mass and intrinsic degrees of stiffness. Together, these form a resonant system, and the mechanical (sonic) resonance may be excited by appropriate sonic frequencies (for example pulses at audio frequencies).

\section{Seismic metamaterials}

Seismic metamaterials, are metamaterials which are designed to counteract the adverse effects of seismic waves on man-made structures, which exist on or near the surface of the earth.

\section{Conclusion}

The utilization of and implementation of MMs at $\mathrm{THz}$ frequencies holds great promise for advancing applications in this technologically relevant region of the EM spectrum.Due to the huge attraction of THz EM MMs , a number of practical MM based THz devices have been developed such as filters, absorbers ,switches and modulators. Though most MM devices operate over a narrow spectral band due to their resonant nature , efforts have been put in making devices with frequency tenability and multiple/ broadband functionality, which 
are favored for applications employing $\mathrm{CW}$ THz source/detectors. The design based on MM have lead to drastic change during last few years. It can be surely stated that the next decades will based on implementation of MMs into real world $\mathrm{THz}$ applications.

\section{References:}

[1] http://en.wikipedia.org/wiki/Metamaterial.

[2] J.B.Pendry, A.J.Holden, D.J.Robbins, and W.J.Stewart, "Magnetism from conductors and enhanced nonlinear phenomena",IEEE Trans.Microw.Theory Tech, vol.47,no.11,pp.2075-2084,Nov.1999.

[3] D.R.Smith,W.J.Padilla,D.C.Vier,S.C.Nemat-Nasser and S.Schultz,"Composite Medium With Simultaneously negative permeability and permittivity",phys.Rev.Lett.,vol.84,pp 4184-4187,May2000.

[4] Shelby, R. A.; Smith, DR; Schultz, S; Smith D.R; Shultz S. (2001). "Experimental Verification of a Negative Index of Refraction". Science 292 (5514): 77-9.]

[5] Guenneau, Sébastien; Alexander Movchan, Gunnar Pétursson, and S. Anantha Ramakrishna (2007). "Acoustic metamaterials for sound focusing and confinement". New Journal of Physics (free download pdf) 9(399): 13672630. Bibcode:2007NJPh...9..399G.doi:10.1088/1367-2630/9/11/399.

[6] Brun, M.; S. Guenneau, and A.B. Movchan (2009-02-09). "Achieving control of in-plane elastic waves". Appl. Phys. Lett. 94 (61903): 1-7. arXiv:0812.0912.Bibcode:2009ApPhL..94f1903B. doi:10.1063/1.3068491.

[7] Wu, B.-I.; W. Wang, J. Pacheco, X. Chen, T. Grzegorczyk and J. A. Kong (2005). "A Study of Using Metamaterials as Antenna Substrate to Ehance Gain"

[8] B.Wood-Metamaterials and its Invisibility.

[9] H. Tao, W.J.Padilla, X.Zhang, and R.D.Averitt -Recent Progress in Electromagnetic Metamaterial Devices for TerahertzApplication 